\title{
CXCR1/2 inhibition enhances pancreatic islet survival after transplantation
}

\author{
Antonio Citro, ${ }^{1,2}$ Elisa Cantarelli, ${ }^{1}$ Paola Maffi, ${ }^{1}$ Rita Nano, ${ }^{1}$ Raffaella Melzi, ${ }^{1}$ Alessia Mercalli, ${ }^{1}$ \\ Erica Dugnani, ${ }^{1}$ Valeria Sordi,, ${ }^{1}$ Paola Magistretti, ${ }^{1}$ Luisa Daffonchio, ${ }^{3}$ Pier Adelchi Ruffini, ${ }^{3}$ \\ Marcello Allegretti, ${ }^{3}$ Antonio Secchi, ${ }^{1,4}$ Ezio Bonifacio, ${ }^{5}$ and Lorenzo Piemonti ${ }^{1}$
}

\begin{abstract}
${ }^{1}$ San Raffaele Diabetes Research Institute (HSR-DRI), S. Raffaele Scientific Institute, Milan, Italy. 2Department of Surgery, University of Pavia, Pavia, Italy. ${ }^{3}$ Research and Development Department, Dompè spa, Aquila, Italy. ${ }^{4}$ Vita Salute San Raffaele University, Milan, Italy. ${ }^{5}$ Center for Regenerative Therapies, Dresden University of Technology, Dresden, Germany.
\end{abstract}

\begin{abstract}
Although long considered a promising treatment option for type 1 diabetes, pancreatic islet cell transformation has been hindered by immune system rejection of engrafted tissue. The identification of pathways that regulate post-transplant detrimental inflammatory events would improve management and outcome of transplanted patients. Here, we found that CXCR1/2 chemokine receptors and their ligands are crucial negative determinants for islet survival after transplantation. Pancreatic islets released abundant CXCR1/2 ligands (CXCL1 and CXCL8). Accordingly, intrahepatic CXCL1 and circulating CXCL1 and CXCL8 were strongly induced shortly after islet infusion. Genetic and pharmacological blockade of the CXCL1-CXCR1/2 axis in mice improved intrahepatic islet engraftment and reduced intrahepatic recruitment of polymorphonuclear leukocytes and NKT cells after islet infusion. In humans, the CXCR1/2 allosteric inhibitor reparixin improved outcome in a phase 2 randomized, open-label pilot study with a single infusion of allogeneic islets. These findings indicate that the CXCR1/2-mediated pathway is a regulator of islet damage and should be a target for intervention to improve the efficacy of transplantation.
\end{abstract}

\section{Introduction}

Achieving long-lasting insulin independence after portal vein islet transplantation has improved, but remains challenging. Nonspecific immune activation (1-6), along with preexisting and transplant-induced auto- and allospecific immune responses (7-9), are components affecting outcome; these are not fully suppressed by ongoing protocols of generalized immunosuppression. Increasing general immunosuppression potency is not ideal because of side effects. Consequently, the development of novel protocols that specifically target proinflammatory immune cell compartments that impede islet function and survival is compelling.

\section{Results and Discussion}

The CXCL1-CXCR1/2 axis is a therapentic target for improving pancreatic islet engraftment. CXCL1 was quantitatively the most relevant inflammatory factor released in culture by mouse islets, both in basal conditions and after IL- $1 \beta$ exposure (Supplemental Figure 1A; supplemental material available online with this article; doi:10.1172/JCI63089DS1). Accordingly, serum CXCL1 concentrations were increased 24 hours after islet infusion into the portal vein (Supplemental Figure 1B), and intrahepatic CXCL1 mRNA expression was strongly increased 4 hours after islet transplantation (>100-fold; Supplemental Figure 1C). CXCL1 signals through the chemokine receptor CXCR2 (10). To investigate the effects of activating the CXCL1-CXCR2 axis in islet engraftment, $\mathrm{Cxcr2} 2^{-1-}$ and $\mathrm{Cxcr}^{+/+}$alloxan-induced severely diabetic BALB-c mice were intrahepatically transplanted with $\mathrm{Cxcr2} 2^{+/+}$syngeneic islets. The probability and median time to reach euglycemia were $62.5 \%$ and

Authorship note: Antonio Citro and Elisa Cantarelli contributed equally to this work. Conflict of interest: Lorenzo Piemonti's research was funded by Dompè Spa in the fiscal year preceding the date of the original submission.

Citation for this article: J Clin Invest. 2012;122(10):3647-3651. doi:10.1172/JCI63089.
4 days, respectively, for $\mathrm{Cxcr}^{+/+}$recipients, compared with $100 \%$ and 1 day for $C x c r 2^{---}$recipients $(P=0.03$; Figure $1 \mathrm{~A})$. The genetically targeted mouse model has some limitations, which include a type 2 inflammatory tendency of the BALB-c mouse (11) and the possible redundancy caused by the binding of CXCL1 to the second recently described murine receptor, CXCR1 (12). We therefore studied the effect of pharmacologic blockade of both CXCR1 and CXCR2 on syngeneic islet engraftment using the type 1 inflammatory-prone C57BL/6 mouse as a recipient. Recipient mice were treated with reparixin, a specific noncompetitive allosteric inhibitor of CXCR1 and CXCR2 (13). Islet engraftment was improved in reparixin- versus vehicle-treated mice when either 150 islet equivalents (IEQs; euglycemia probability, $84 \%$ vs. $50 \%$; median time to euglycemia, 7 vs. 21 days; $P=0.04$ ) or 250 IEQs (euglycemia probability, $100 \%$ vs. $57 \%$; median time to glycemia, 1 vs. 50 days; $P=0.002$ ) were used for transplant (Figure 1A).

CXCR1/2 inbibitor significantly reduces postinfusion recruitment of PMNs and NKT cells. Among intrahepatic leukocyte (IHL) populations, CXCR2 was expressed on all polymorphonuclear leukocytes (PMNs; Gr1 ${ }^{+} \mathrm{CD} 11 \mathrm{~b}^{+} \mathrm{Ly} 6 \mathrm{C}^{-} ; 97 \pm 1 \%$ ) and on a subpopulation of NKT cells (NK1.1 ${ }^{+} \mathrm{CD}^{+} ; 25 \pm 6 \%$ ) (Figure 1B). Both cell populations increased markedly in the livers of mice transplanted intrahepatically with syngeneic or allogeneic islets, and treatment of mice with the CXCR $1 / 2$ inhibitor significantly inhibited this rise (Figure 1C and Supplemental Table 1). Other IHL populations were only marginally affected by reparixin treatment (data not shown). The identification of a targetable pathway able to prevent the recruitment of both these cells is an important finding. PMNs are the predominant cell type infiltrating the islets in experimental models (14), and a vicious cycle mediated by NKT and PMN IFN- $\gamma$-based cross-talk was recently demonstrated to be harmful to transplanted islets (15). Concordantly, NKT cell inhibition or deletion improves engraftment and delays islet rejection $(1,2,15)$. 

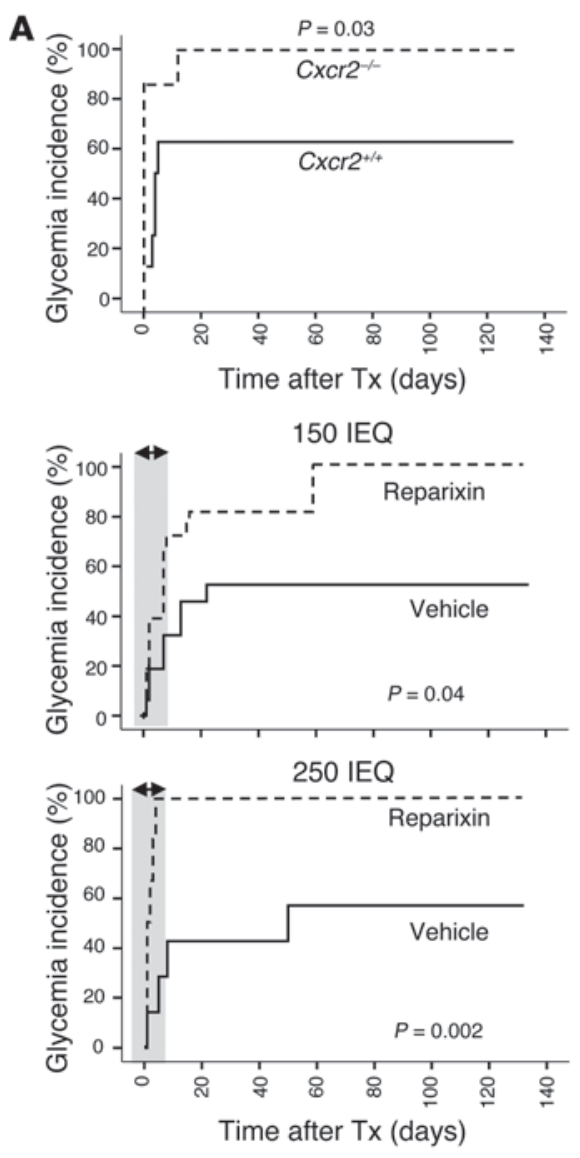

C
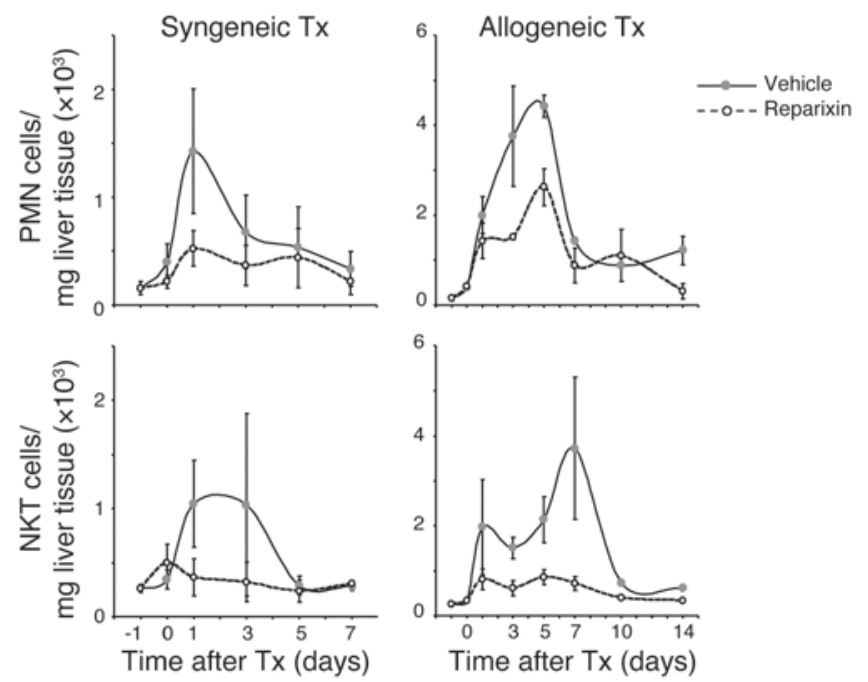

Figure 1

Genetic and pharmacologic blockade of the CXCL1-CXCR1/2 axis. (A) Kaplan-Meier analysis for achieving normoglycemia (<200 mg/dl) in $\mathrm{CxCr}^{-/-}(n=7)$ and $\mathrm{Cxcr}^{+/+}(n=8)$ alloxan-induced severely diabetic BALB-c mice transplanted with $350 \mathrm{Cxcr2^{+/+ }}$ syngeneic islets through the portal vein. Also shown is achievement of normoglycemia in alloxan-treated severely diabetic C57BL/6 mice transplanted (Tx) with 150 IEQs $(n=46)$ or 250 IEQs $(n=17)$ through the portal vein in the presence of reparixin $(n=25$ [150 IEQs]; 9 [250 IEQs]) or vehicle $(n=21$ [150 IEQs]; 8 [250 IEQs]). Differences were tested using log-rank statistic. The treatment window is shaded gray. (B) CXCR2 expression on IHLs from untreated control mice measured by flow cytometry 5 days after islet transplantation. (C) C57BL/6 recipients were transplanted with 400 C57BL/6 syngeneic or BALB-c allogeneic IEQs into the portal vein in the presence of either reparixin or vehicle. 6 mice ( 3 reparixin; 3 vehicle) were analyzed per time point. IHL populations were analyzed by flow cytometry. Data are mean \pm SEM. For statistical comparisons, see Supplemental Table 1.

Pharmacological blockade of CXCR1/2 improves islet transplantation outcome in humans. Chemokine and cytokine release was examined after 24 hours of culture of 44 human islet preparations (Figure 2). CXCL8, the human functional homolog of mouse CXCL1, was quantitatively the most relevant inflammatory factor released. Serum cytokine/chemokine concentrations were analyzed in 11 patients with type 1 diabetes who received allogeneic islet transplants under 2 immunosuppression regimens: ATG, FK506, and MMF $(n=3$; NCT01220856), or ATG, RAPA, and MMF plus steroid and IL-1 receptor antagonist $(n=8$; NCT01346085). CXCL8 significantly increased after islet infusion in both groups, which suggests that its release is not fully controlled by standard imunosuppression protocols that include antiinflammatory agents.

The findings in mouse and humans led to a phase 2 randomized, open-label pilot study to assess the efficacy and safety of CXCR1/2 inhibition after single-infusion islet transplantation. Inclusion criteria restricted enrollment to patients expected to receive an islet mass in the lower range of the currently accepted transplantable islet amount. Patients were placed on an immunosuppression regi- men consisting of induction with ATG and maintenance immunosuppression with FK506 and MMF. Patients were randomly assigned to receive either no additional experimental intervention or CXCR1/2 inhibitor reparixin treatment $(2.772 \mathrm{mg} / \mathrm{kg} \mathrm{BW} / \mathrm{h}$ i.v. continuous infusion for 7 days starting from day -1$)$. Initially, 7 consecutive patients were enrolled, with randomization to reparixin treatment in 4 patients (Pt2, Pt3, Pt5, and Pt7; see Supplemental Table 2 and Table 1 for islet and patient characteristics). No reparixin-related adverse events were reported in these patients. None of the patients in the control group expressed $\beta$ cell function 1 month after transplant (no decrease in insulin requirement coupled with C-peptide levels less than $0.09 \mathrm{ng} / \mathrm{ml}$ ), and all were withdrawn as a result of graft loss. In contrast, patients treated with reparixin experienced improved transplant outcome, as measured by glycemic control, decreased insulin requirement, and appearance of detectable levels of C-peptide well above $0.3 \mathrm{ng} / \mathrm{ml}$. Fasting C-peptide, reduction of insulin requirement, stimulated C-peptide (evaluated as peak and area under the curve response derived from the 2-hour mixed-meal tolerance test; ref. 16), and islet estimated function (17) 
A

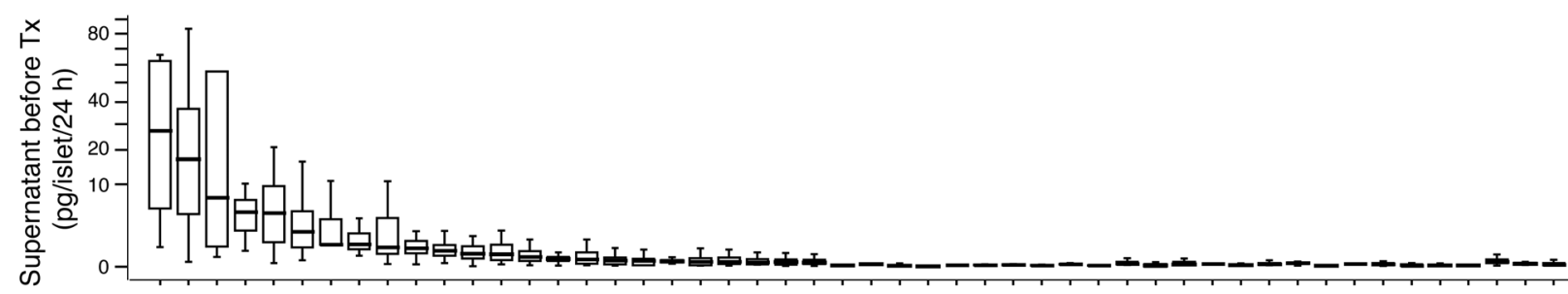

B

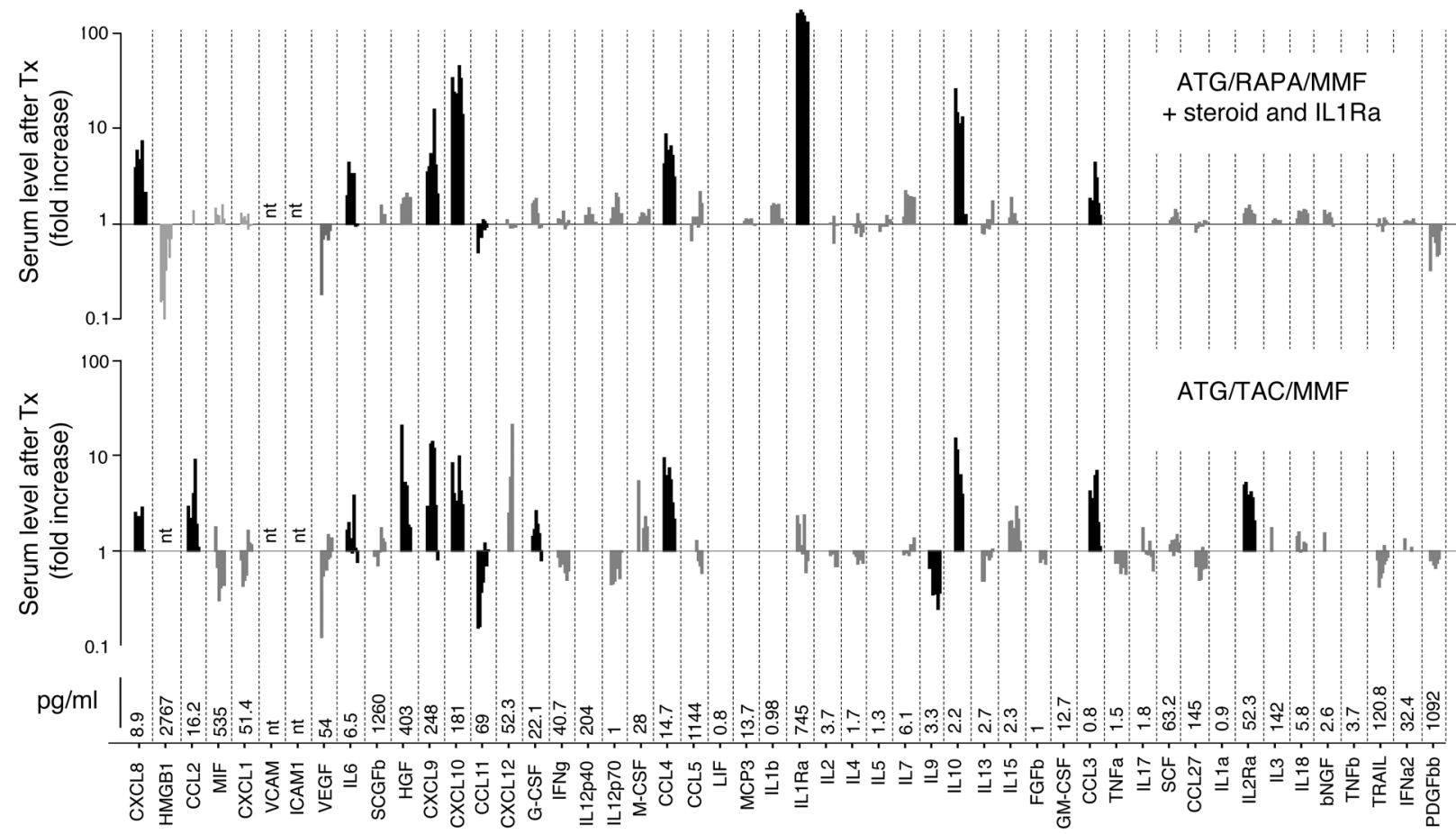

\section{Figure 2}

Inflammatory status before and after allogeneic islet transplantation in patients with type 1 diabetes. (A) Human islets (1,000 IEQ/ml) from 44 pancreas preparations processed from February 2007 to June 2010 at San Raffaele Scientific Institute (19 male, 25 female; age, $50 \pm 14$ years; $\mathrm{BMI}, 26.3 \pm 3.8$; purity, $50 \% \pm 24 \%$ ) were cultured before transplantation, and chemokine/cytokine concentrations after 24 hours were measured in the supernatants. Boxes denote interquartile range; lines within boxes denote median; whiskers denote $5 \%$ and $95 \%$ limits. (B) Circulating cytokine/chemokine concentrations were evaluated 1, 3, 6, 12, 24, 72, 120, and 168 hours after islet infusion in 11 patients with type 1 diabetes receiving islet transplantation alone at San Raffaele Scientific Institute under 2 protocols: ATG plus low-dose steroids plus IL-1 receptor antagonist (IL1Ra) and (as maintenance) immunosuppression with rapamycin plus MMF ( $n=8)$; and ATG and (as maintenance) immunosuppression with FK506 plus MMF $(n=3)$. Data are expressed as median fold increase versus basal at each time point. Mean basal concentrations are shown below. Statistical analysis was performed by Mann-Whitney $U$ test (black bars, $P \leq 0.05$; gray bars, $P=N S$ ). nt, not tested.

were all significantly higher in the reparixin group than in controls (Figure 3). Based on these results, the ethical review board recommended protocol amendment allowing randomization to the reparixin treatment group only and to allow patients with a functioning graft to receive a second islet infusion. 2 additional patients (Pt8 and Pt9) received islets with reparixin treatment. Pt8 demonstrated excellent islet function (Figure 3), and Pt9 did not comply with oral immunosuppression treatment and dropped out of the study. The one reparixin-treated patient who did not have markedly improved transplant outcome, Pt7, had a 10-fold increase in GAD autoantibody titer soon after transplant. Pt2, Pt3, and Pt5 from the initial reparixin treatment group received a second islet infusion, and Pt 2 and $\mathrm{Pt} 3$ achieved insulin independence (total infused, 7,589 and 8,888 IEQ/kg, respectively).

With the exception of selective CCR5 antagonists for HIV, our results represent the first clinical evidence to our knowledge that chemokine receptor blockade may have therapeutic benefit. Although the numbers in the trial were small, we demonstrated that an important cause of transplanted islet dysfunction in humans was nonspecific inflammation mediated by CXCR $1 / 2$ receptor, which is inadequately inhibited by ongoing protocols of generalized immunosuppression. Collectively, the findings suggest a novel approach to prevent early loss of transplanted islets. A phase 3 multicenter, randomized, double-blind, parallel-assignment trial has commenced to confirm the results of this pilot study. We further suggest that targeting the CXCR1/2 pathway may be therapeutically relevant for the preservation of islet function in new-onset type 1 diabetes.

\section{Methods}

Mice. Male C57BL/6 and BALB-c mice were purchased from Charles River Laboratories. Cxcr2- $2^{--}$mice on BALB-c background [strain C129S2 (B6)- 


\section{brief report}

Table 1

Characteristics of recipients in the reparixin islet transplant trial: first infusion

\begin{tabular}{|c|c|c|c|c|c|c|c|c|c|c|}
\hline Patient & $\begin{array}{l}\text { Age } \\
\text { (yr) }\end{array}$ & Sex & $\begin{array}{c}\text { Diabetes } \\
\text { duration (yr) }\end{array}$ & $\begin{array}{l}\text { Weight } \\
\text { (kg) }\end{array}$ & BMI & $\begin{array}{l}\text { Insulin } \\
\text { (U/kg/d) }\end{array}$ & HbA1c & $\begin{array}{l}\text { Follow-up } \\
\text { (d) }\end{array}$ & $\begin{array}{c}\text { C-pep } \\
>0.09 \mathrm{ng} / \mathrm{ml}\end{array}$ & $\begin{array}{l}\text { Treatment } \\
\text { withdrawn }\end{array}$ \\
\hline \multicolumn{11}{|l|}{ Controls } \\
\hline Pt1 & 56 & $\mathrm{~F}$ & 41 & 50 & 20.8 & 0.33 & 7.9 & 318 & Day 0-36 & $59 d$ \\
\hline Pt4 & 45 & M & 28 & 67 & 23.5 & 0.49 & 7.1 & 270 & Day 0-27 & $76 \mathrm{~d}$ \\
\hline Pt6 & 43 & $F$ & 34 & 58.5 & 20.2 & 0.72 & 8.7 & 222 & Day 0-26 & $51 \mathrm{~d}$ \\
\hline Total & $48 \pm 4$ & & $33.6 \pm 4$ & $58.5 \pm 4.9$ & $21.5 \pm 1$ & $0.51 \pm 0.11$ & $7.9 \pm 0.46$ & & & \\
\hline \multicolumn{11}{|c|}{ Reparixin } \\
\hline Pt2 & 40 & M & 21 & 60 & 21.5 & 0.38 & 9.8 & 280 & Day 0-280 & No \\
\hline Pt3 & 49 & $\mathrm{~F}$ & 45 & 47 & 21.5 & 0.72 & 9.1 & 310 & Day 0-310 & No \\
\hline Pt5 & 42 & $M$ & 22 & 69 & 23.1 & 0.48 & 9.8 & 230 & Day $0-230$ & No \\
\hline Pt7 & 63 & $M$ & 34 & 62 & 22.8 & 0.89 & 6.7 & 151 & Day 0-102 & $56 \mathrm{~d}$ \\
\hline Total & $48.5 \pm 5$ & & $30.5 \pm 6$ & $59.5 \pm 4.5$ & $22.2 \pm 0.4$ & $0.61 \pm 0.11$ & $8.8 \pm 0.73$ & & & \\
\hline \multicolumn{11}{|c|}{ After protocol amendment } \\
\hline Pt8 & 46 & $\mathrm{~F}$ & 12 & 60 & 22 & 0.92 & 9.7 & 124 & Day $0-124$ & No \\
\hline Pt9 & 41 & $\mathrm{~F}$ & 4 & 47 & 18.8 & 0.74 & 9.7 & Drop out & Drop out & Drop out \\
\hline
\end{tabular}

IL8RB] were provided by Dompè spa. Pancreatic islets were isolated and transplanted via the portal vein as previously described (11). Reparixin was provided by Dompè spa and administered by s.c. continuous infusion (Alzet Osmotic pump; Alza Corp.) starting from day -1 up to day 6 after islet transplantation at a dose of $5.4 \mathrm{mg} / \mathrm{kg} / \mathrm{h}$. Recipient mice were ren- dered diabetic with i.v. alloxan injection $(60-75 \mathrm{mg} / \mathrm{kg}$; Sigma-Aldrich). Blood glucose measurements were performed using a Glucometer Elite (Bayer Canada). Euglycemia was defined as nonfasting blood glucose concentrations of less than $200 \mathrm{mg} / \mathrm{dl}$ in 2 consecutive measurements. For evaluation of intrahepatic leukocytes, single-cell suspensions were pre-
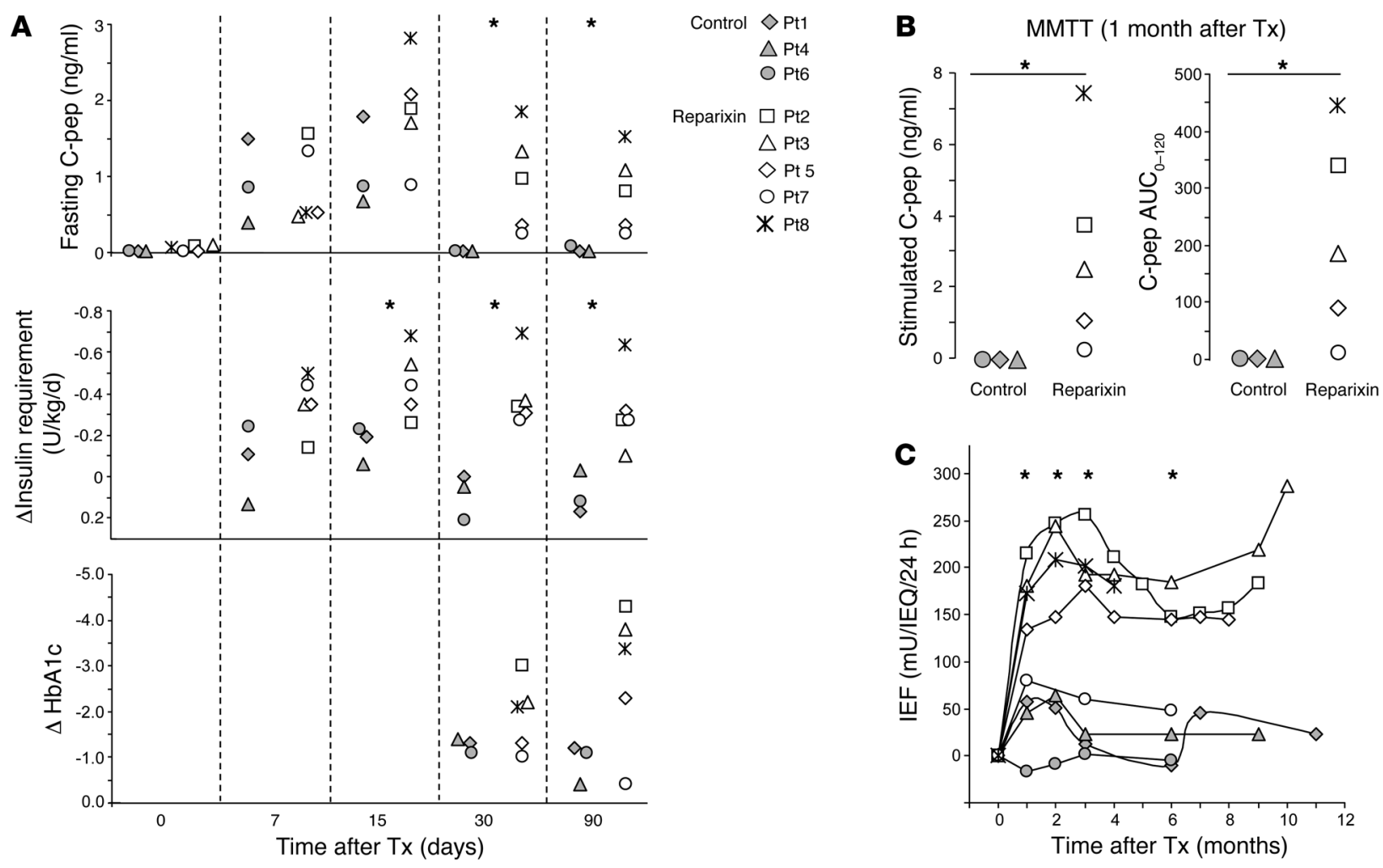

Figure 3

Reparixin treatment influences allogeneic islet transplantation outcome in patients with type I diabetes. (A) Individual patients during 3 months of follow-up after single islet transplantation for patients receiving reparixin and controls without reparixin treatment. Shown are fasting C-peptide, change in average daily insulin requirement, and $\mathrm{HbA1c}$ (expressed as an absolute decrease from pretransplant values). (B) Mixed-meal tolerance test (MMTT) 1 month after islet infusion. MMTT was performed after an overnight fast, as described by Greenbaum (16). Maximum and area under the curve (AUC) of C-peptide is shown. (C) Individual islet estimated function (IEF; ref. 17) after single islet transplantation in the presence or absence of reparixin treatment. All comparisons were made using Mann-Whitney test. ${ }^{\star} P \leq 0.05$. 
pared from 2 liver lobes of known weight, and analysis of IHL population was performed by flow cytometry. Cells were stained with FITC-, PE-, or allophycocyanin-labeled (APC-labeled) anti-CD4, anti-CD8, anti-CD3, anti-CD19, anti-TCRb, anti-NK1.1, anti-CD11, anti-Gr-1, anti-CD11b, anti-Ly6C, or anti-CD11c Abs (BD Biosciences - Pharmingen).

Human islet transplantation. Circulating cytokine/chemokine concentrations after islet infusion were measured in 11 patients with type 1 diabetes receiving islet transplantation alone at San Raffaele Scientific Institute. 8 recipients (NCT01346085) were treated with: (a) pretransplant rapamycin $(0.1 \mathrm{mg} / \mathrm{kg} / \mathrm{d}$, targeting serum trough levels of $8-10 \mathrm{ng} / \mathrm{ml})$ for at least 30 days prior to first islet infusion; (b) ATG induction therapy $(1.5 \mathrm{mg} /$ $\mathrm{kg} / \mathrm{d}$ for 4 days) and a steroid bolus (methyl-prednisolone, $500 \mathrm{mg}$ bolus) plus low-dose steroids (prednisone, $10 \mathrm{mg} / \mathrm{d}$ ) and Anakirna $(100 \mathrm{mg} / \mathrm{d}$ ) for 2 weeks; (c) maintenance immunosuppressive therapy with rapamycin $(0.1 \mathrm{mg} / \mathrm{kg} / \mathrm{d}$, targeting serum trough levels of $12-15 \mathrm{ng} / \mathrm{ml})$ plus MMF $(2 \mathrm{~g} / \mathrm{d}) .3$ recipients (NCT01220856) were treated with: (a) ATG induction therapy $(1.5 \mathrm{mg} / \mathrm{kg} / \mathrm{d}$ for 4 days) and a steroid bolus (methyl-prednisolone, $500 \mathrm{mg}$ bolus); (b) maintenance immunosuppressive therapy with FK506 (targeting serum trough levels of 8-10 ng/ml) plus MMF $(2 \mathrm{~g} / \mathrm{d})$. The characteristics of islet preparations, donors, and recipients are reported in Supplemental Table 3.

Reparixin in pancreatic islet transplantation trial. A phase 2 randomized, open-label, pilot study to assess the efficacy and safety of reparixin after single-infusion islet transplantation in patients with type 1 diabetes mellitus was initiated in July 2010 (NCT01220856). Inclusion criteria restricted enrolment to patients expected to receive an islet mass in the lower range of the currently accepted transplantable islet amount (3,000-7,000 IEQ/kg BW). Patients were on an immunosuppression regimen consisting of: (a) ATG induction therapy $(1.5 \mathrm{mg} / \mathrm{kg} / \mathrm{d}$ for 4 days starting at day -1) and a steroid bolus (methyl-prednisolone, $500 \mathrm{mg}$ bolus, day -1), (b) maintenance immunosuppressive therapy with mycophenolate mofetil ( $2 \mathrm{~g} / \mathrm{d}$ starting on day -1 of islet infusion) plus tacrolimus $(0.087 \mathrm{mg} / \mathrm{kg}$ twice daily, targeting serum trough levels of $8-10$ $\mathrm{ng} / \mathrm{ml})$ replaced by rapamycin from month 3 after transplant $(0.1 \mathrm{mg} /$ $\mathrm{kg} / \mathrm{d}$, targeting serum trough levels of $10-12 \mathrm{ng} / \mathrm{ml}$ ). Patients were randomly (1:1) assigned to receive either no additional experimental inter- vention (control group) or reparixin treatment $(2.772 \mathrm{mg} / \mathrm{kg} \mathrm{BW} / \mathrm{h}$ i.v. continuous infusion for 7 days from day -1).

Basal release of chemokines and cytokines in freshly isolated mouse and human islets. Human and mouse cytokines/chemokines were detected using multiplex bead-based assays (Bio-Plex Human Cytokine 27-Plex Panel; BioPlex Human Group II Cytokine 23-Plex Panel; Bio-Plex Mouse Cytokine 23-Plex Panel; Biorad Laboratories).

Statistics. Analysis of data was performed using the SPSS statistical package for Windows (SPSS Inc.). A 2 -tailed $P$ value less than 0.05 was considered significant.

Study approval. All patients gave informed consent for the investigations. The ethical committee of the Istituto Scientifico Ospedale San Raffaele approved the islet transplant protocols and investigations. Investigations were carried out in accordance with the principles of the Declaration of Helsinki, as revised in 2000. All mouse experiments were in accordance with protocols approved by the Animal Care and Use Committee of San Raffaele Scientific Institute.

\section{Acknowledgments}

This work was supported by the EU (HEALTH-F5-2009-241883BetaCellTherapy), the Juvenile Diabetes Research Foundation (JDRF grants 6-2006-1098, 31-2008-416, and 4-2001-434), and a research grant from Dompé spa to L. Piemonti (HSR-DRI, Milan). C. Antonio is enrolled as a PhD student in the Department of Surgery at University of Pavia. E. Cantarelli conducted this study as partial fulfillment of her PhD in Molecular Medicine, Program in Basic and Applied Immunology, San Raffaele University. The phase 2 clinical trial is sponsored by Dompé spa.

Received for publication January 27, 2012, and accepted in revised form August 2, 2012.

Address correspondence to: Lorenzo Piemonti, San Raffaele Diabetes Research Institute (HSR-DRI), S. Raffaele Scientific Institute, Via Olgettina 60, 20132 Milan, Italy. Phone: 39.02.26432706; Fax: 39.02.26432871; E-mail: piemonti.lorenzo@hsr.it.
1. Toyofuku A, et al. Natural killer T-cells participate in rejection of islet allografts in the liver of mice. Diabetes. 2006;55(1):34-39.

2. Yasunami Y, et al. Valpha14 NK T cell-triggered IFN-gamma production by Gr-1+CD $11 b+$ cells mediates early graft loss of syngeneic transplanted islets. J Exp Med. 2005;202(7):913-918.

3. Moberg L, et al. Production of tissue factor by pancreatic islet cells as a trigger of detrimental thrombotic reactions in clinical islet transplantation. Lancet. 2002;360(9350):2039-2045.

4. Johansson $\mathrm{H}$, et al. Tissue factor produced by the endocrine cells of the islets of Langerhans is associated with a negative outcome of clinical islet transplantation. Diabetes. 2005;54(6):1755-1762.

5. Melzi R, et al. Role of CCL2/MCP-1 in islet transplantation. Cell Transplant. 2010;19(8):1031-1046.

6. Piemonti L, et al. Human pancreatic islets produce and secrete MCP-1/CCL2: relevance in human islet transplantation. Diabetes. 2002;51(1):55-65.

7. Monti $\mathrm{P}$, et al. Islet transplantation in patients with autoimmune diabetes induces homeostatic cytokines that expand autoreactive memory T cells. J Clin Invest. 2008;118(5):1806-1814.

8 . Hilbrands R, et al. Differences in baseline lymphocyte counts and autoreactivity are associated with differences in outcome of islet cell transplantation in type 1 diabetic patients. Diabetes. 2009; 58(10):2267-2276.

9. Huurman VA, et al. Cellular islet autoimmunity associates with clinical outcome of islet cell transplantation. PLoS One. 2008;3(6):e2435.

10. Lee J, Cacalano G, Camerato T, Toy K, Moore MW, Wood WI. Chemokine binding and activities mediated by the mouse IL-8 receptor. J Immunol. 1995;155(4):2158-2164.

11. Melzi R, Sanvito F, Mercalli A, Andralojc K, Bonifacio E, Piemonti L. Intrahepatic islet transplant in the mouse: functional and morphological characterization. Cell Transplant. 2008;17(12):1361-1370.

12. Fu W, Zhang Y, Zhang J, Chen WF. Cloning and characterization of mouse homolog of the CXC che- mokine receptor CXCR1. Cytokine. 2005;31(1):9-17.

13. Bertini R, et al. Noncompetitive allosteric inhibitors of the inflammatory chemokine receptors CXCR1 and CXCR2: prevention of reperfusion injury. Proc Natl Acad Sci U S A. 2004;101(32):11791-11796.

14. Moberg L, Korsgren O, Nilsson B. Neutrophilic granulocytes are the predominant cell type infiltrating pancreatic islets in contact with $\mathrm{ABO}$-compatible blood. Clin Exp Immunol. 2005;142(1):125-131.

15. Matsuoka $\mathrm{N}$, et al. High-mobility group box 1 is involved in the initial events of early loss of transplanted islets in mice. J Clin Invest. 2010; 120(3):735-743.

16. Greenbaum C, Seidel K, Pihoker C. The case for intravenous arginine stimulation in lieu of mixedmeal tolerance tests as outcome measure for intervention studies in recent-onset type 1 diabetes. Diabetes Care. 2004;27(5):1202-1204.

17. Caumo A, et al. Transplant estimated function: a simple index to evaluate beta-cell secretion after islet transplantation. Diabetes Care. 2008;31(2):301-305. 